\title{
Update in Vitreoretinal Instrumentation
}

\author{
Gerardo González-Saldivar and David R Chow \\ Department of Ophthalmology, University of Toronto, St. Michael's Hospital, Toronto, Canada
}

DOl: https://doi.org/10.17925/USOR.2018.11.2.98

$\mathrm{O}$

ver the past 45 years, vitreoretinal surgery has evolved considerably. Today, retinal surgeons have access to a wide variety of instruments for micro-incision vitrectomy surgery, which will result in more comfortable, safer, and efficacious surgery. We aim to expose some of the most relevant innovations for vitreoretinal surgery.

\section{Keywords}

Vitreoretinal instrumentation,

surgery, innovations, retina

Disclosures: Gerardo González-Saldivar has nothing to declare in relation to this article. David R Chow declares the following financial interests or relationships: consultant/advisor for Alcon Laboratories, Inc. Bayer Healthcare Pharmaceuticals, Dutch Ophthalmic Research Center (DORC) International USA and Katalyst; lecture fees for DORC International USA, OptoVue and Allergan; and equity ownership/stock options in public or privately traded firms, excluding mutual funds from Katalyst and Synergetics, Inc.

Review Process: Double-blind peer review.

open Access Statement: This article is published under the Creative Commons Attribution Noncommercial License, which permits any non-commercial use, distribution, adaptation, and reproduction provided the original author(s) and source are given appropriate credit. ( ) The Authors 2018.

Authorship: All named authors meet the criteria of the International Committee of Medical Journal Editors for authorship for this manuscript, take responsibility for the integrity of the work as a whole, and have given final approval for the version to be published.

Received: June 21, 2018

Accepted: August 13, 2018

Citation: US Ophthalmic Review. 2018;11(2):98-102

Corresponding Author: Gerardo González-Saldivar, St. Michael's Hospital, University of Toronto, Retina Department, Toronto, Canada. E: dr.gerardo@meduanl.com

Support: No funding was received in the publication of this article.
Over the past 45 years, vitreoretinal surgery has evolved considerably. In 1972, Machemer et al. introduced the concept of pars plana vitrectomy with a 17-gauge vitreous cutter.' Today, retinal surgeons have access to a wide variety of instruments as small as 27-gauge for micro-incision vitrectomy surgery. Progress has occurred not only in vitrectomy probes, which tend to be smaller, faster, and safer, but in all other aspects of our core instrumentation.

Recent developments include new techniques for endoillumination, handheld instruments and forceps, wound construction, and flexible laser probes. Knowing the peculiarities and possible specific uses for these new instruments increases the possibility of obtaining excellent outcomes in vitreoretinal surgery. On the other hand, novel visualization technologies such as the "3D heads up" viewing system and the adaptation of intraoperative optical coherence tomography (iOCT) continue to improve existing viewing modalities: innovations that will result in more comfortable, safer, and efficacious surgery.

\section{Cutters}

One of the most important instruments in vitreoretinal surgery is the cutter. This device allows effective removal of the hyaloid and vitreous base, core vitrectomy, and membrane cutting. Factors that have been modified in order to improve this instrument and reduce retinal traction are the blade design, the duty cycle, the cutting speed, and the tip-to-port distance. ${ }^{2}$

\section{EVA Two Dimensional Cutter}

The Dutch Ophthalmic Research Center (DORC) has developed the Two Dimensional Cutter for their EVA phaco-vitrectomy system (DORC Dutch Ophthalmic Center [International] BV, Zuidland, The Netherlands). This features a larger aperture port with two cutting openings in the guillotine shaft that cuts vitreous in both the forward and backward direction of the probe (Figure 1). No matter the position of the blade, the port is never completely occluded. This allows cut rates of up to $16,000 \mathrm{cpm}$ and a $92 \%$ duty cycle that creates constant aspiration flow.

The advantages of this blade design are the twofold increase in cut rates, increased flow rates, and decreased retinal traction. The Two Dimensional Cutter has shown greater performance removing thick membranes and lens fragments due to its $42 \mathrm{psi}$ ( $290 \mathrm{kPa}$ ) working pressure even with 27-gauge instrumentation. Cutters are available in 23-, 25-, and 27-gauge. ${ }^{3}$

\section{Constellation}

Three major modifications have been made to the Constellation cutters (Alcon, Fort Worth, TX, US), which include increased speed, a better blade design, and a shorter tip-to-port distance. Constellation's newest cutters (Ultravit ${ }^{\circledR}$ High Speed Vitrectomy Probe) perform up to 10,000 cpm, achieving this by dual pneumatic drive technology. This increased cutter speed may minimize turbulence and decrease peripheral retinal traction while trimming the vitreous base. This platform also modified the cutter tip; by beveling the tip (Figure 2) they decreased the tip-to-port distance to 0.009 inches $(0.23 \mathrm{~mm})$ (Table 1). This allows surgeons to access tighter tissue planes. All modifications are available in 23-, $25-$, and 27 -gauge series. ${ }^{4}$ 
Figure 1: EVA Two Dimensional Cutter

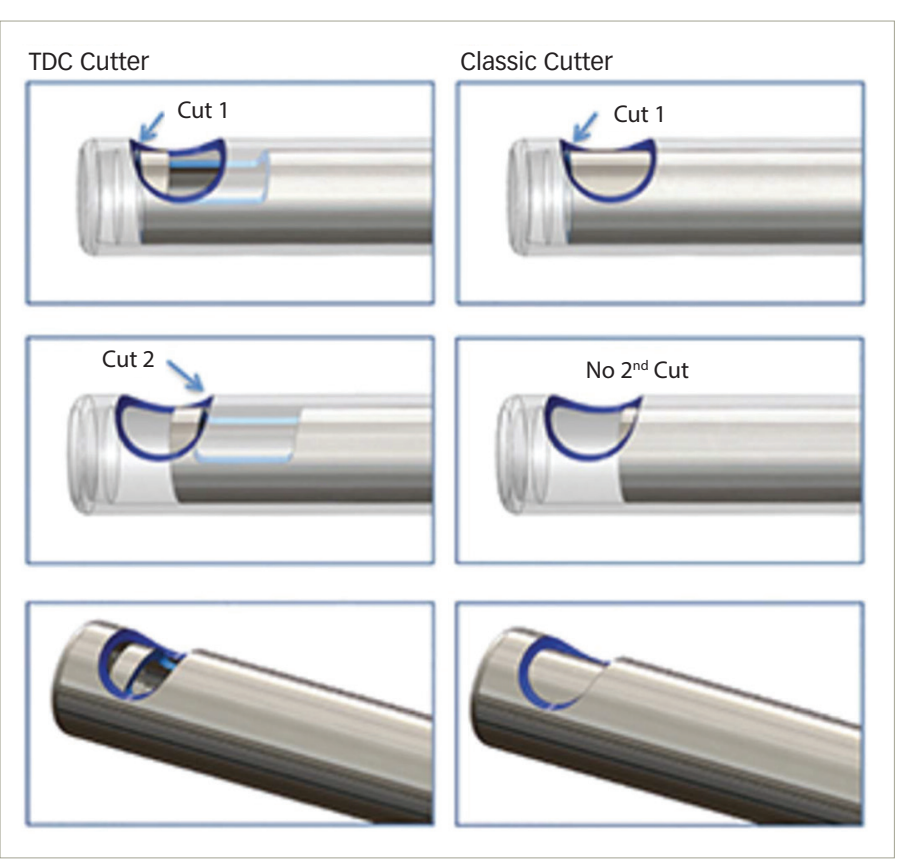

EVA Two Dimensional Cutter (Dutch Ophthalmic Research Center [International] BV, Zuidland, The Netherlands).

Figure 2: Tip-to-port distance enables the beveled cutter port to be closer to the retina

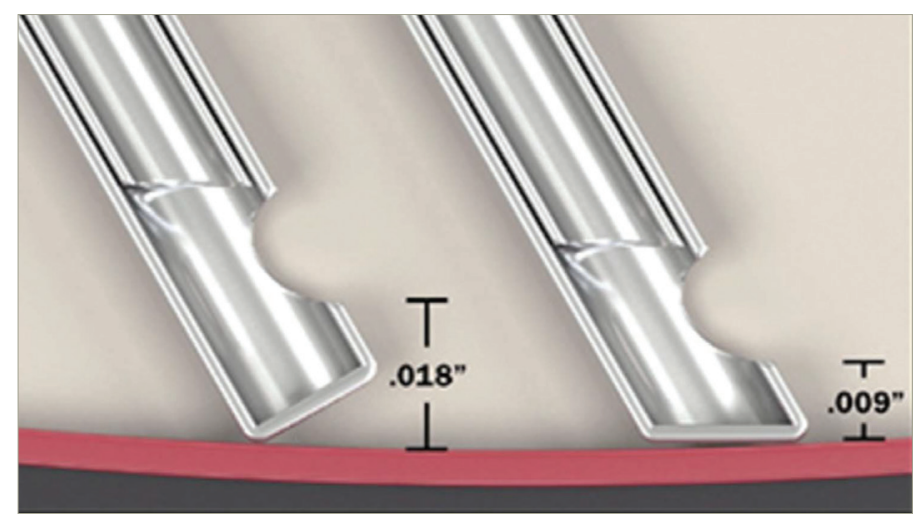

Constellation Ultravit ${ }^{\circledR}$ High Speed Vitrectomy Probe (Alcon, Fort Worth, TX, US).

Table 1: Tip-to-port distance in Alcon standard and beveled-tip probes

\begin{tabular}{|l|l|l|l|}
\hline Probe & Standard tip (inches) & Beveled tip (inches) & $\%$ \\
\hline 23-gauge & 0.020 & 0.009 & 55 \\
\hline 25-gauge & 0.017 & 0.009 & 47 \\
\hline 27-gauge & 0.015 & 0.009 & 40 \\
\hline
\end{tabular}

$\%=$ reduction of the distance between the cutting port and retina.

The latest blade design that has been implemented for the Constellation is the Hypervit vitrectomy probe. This probe has a dual-blade mechanism (two cuts per duty cycle) achieving up to 20,000 cpm, reducing retinal traction, and allowing consistent flow rates. It also has a beveled-tip design and is available in 23-, 25-, and 27-gauge series. ${ }^{4}$
Figure 3: Vitesse hypersonic vitrectomy

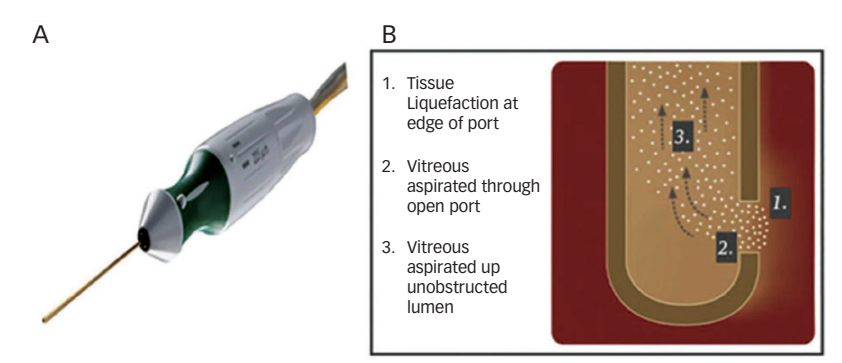

A) ocutome; B) mechanism of action. Vitesse Hypersonic Vitrectomy probe (Bausch + Lomb Retina, St Louis, MO, US).

Figure 4: Auto inserter

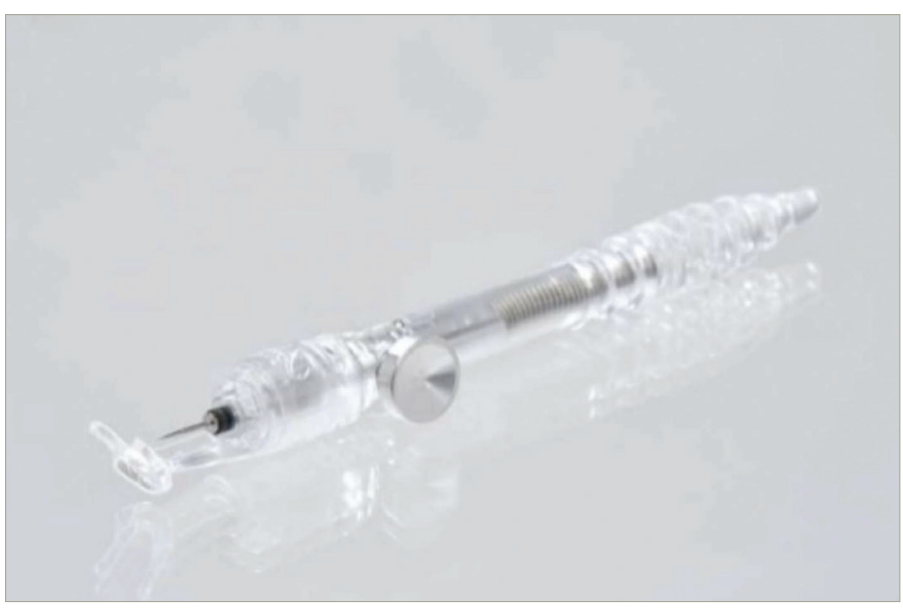

Auto inserter (Bausch + Lomb, Rochester, NY, US).

\section{Stellaris Elite}

The Stellaris Elite ${ }^{\mathrm{TM}}$ system (Bausch + Lomb Retina, St Louis, MO, US) has introduced two new cutters for their platform. The Bi-Blade design features a dual-cutting action for each duty cycle due to the inner needle design, increasing the effective cut rate from 7,500 cpm to $15,000 \mathrm{cpm}$. Since the cutter port is open almost all of the time, the flow rates achieved with this cutter increase dramatically.

The most novel vitrectomy probe to enter the market in the past few years is the Vitesse Hypersonic Vitrectomy probe (Bausch + Lomb Retina, St Louis, MO, US), which is exclusively compatible with the Stellaris Elite platform (Figure 3). Hypersonic vitrectomy is based on a piezoelectric ultrasound transducer which liquefies the vitreous at the edge of the port where it is then aspirated. The hypersonic vitrector tip pulsates at 1.7 million times per minute, and has a 100\% open port vitrectomy system which allows high and constant flow rates. Early clinical usage has suggested that the Vitesse ultrasound transducer may allow removal of silicone oil with this probe, a feature not associated with other traditional vitrectomy cutters. ${ }^{4}$

\section{Auto inserter}

The auto inserter (Bausch + Lomb, Rochester, NY, US) is a device employed for the automated insertion of scleral cannulae instead of the conventional manual method (Figure 4). The automated insertion has been demonstrated 
Figure 5: Graph demonstrates the amount of pressure required to puncture the globe

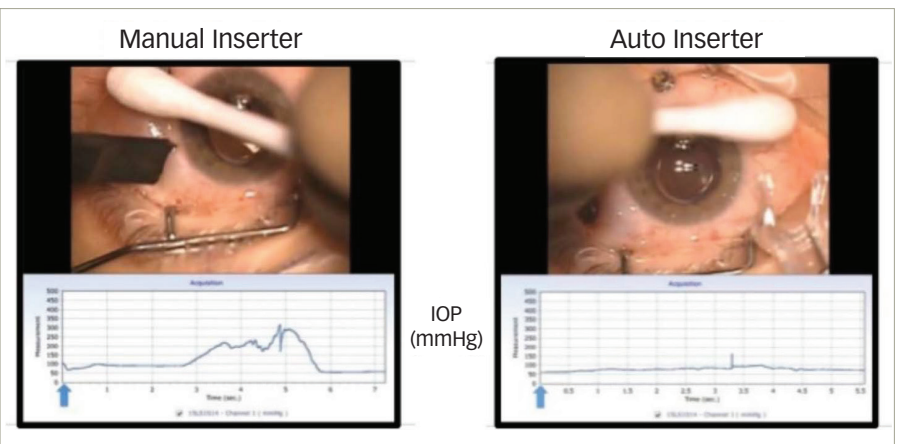

$1 O P=$ intraocular pressure

Figure 6: Vektor Articulating Illuminated Laser Probe

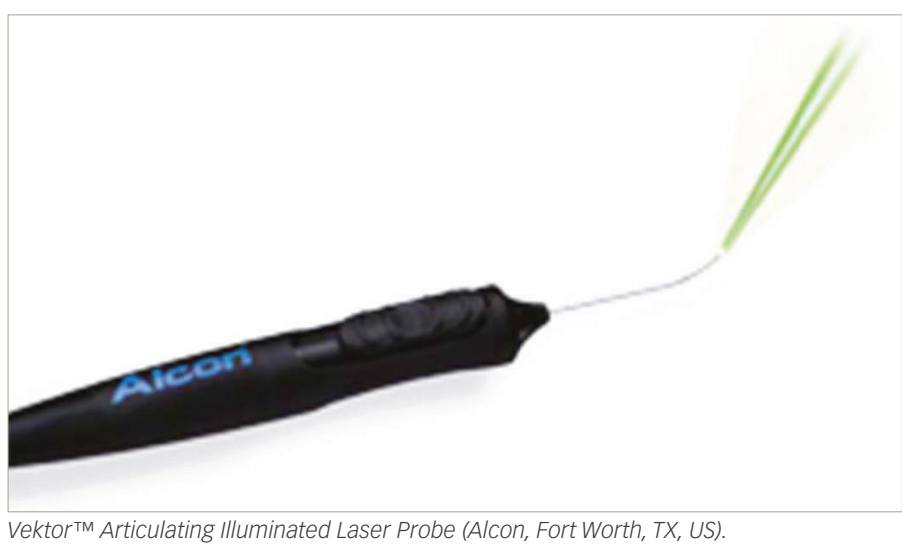

to significantly decrease the amount of pressure required in order to puncture the globe (Figure 5). This might be a great solution for soft eyes, as very little pressure is needed for their insertion.

\section{Vektor Articulating Illuminated Laser Probe}

The Vektor ${ }^{\mathrm{TM}}$ Articulating Illuminated Laser Probe (Alcon, Fort Worth, TX, US) is a new illuminated laser probe with an articulating tip (Figure 6). It is effective in providing an optimal scraping force and minimal retinal trauma. ${ }^{4}$

\section{FINESSE Flex Loop}

The FINESSETM nitinol (nickel/titanium) Flex Loop (Alcon, Fort Worth, TX, US) is effective in providing an optimal scraping force and minimal retinal trauma. The thin-loop design allows excellent visibility of the contact area and its retractable tip allows adjustable lengthening of the loop as a consequence of its stiffness. Finesse loop has been employed for epiretinal membrane (ERM) dissection, internal limiting membrane (ILM) peeling, and proliferative vitreoretinopathy membrane removal. It is available in 23-, 25-, and 27- gauge (Figure 7). ${ }^{4}$

\section{D printer customized retinal forceps}

since modern forceps are disposable and mass produced, there are many variables that are not taken into consideration when developing their handle because they are produced at standard measurements. Some of these variables are the size and shape of a surgeon's hand and grip.

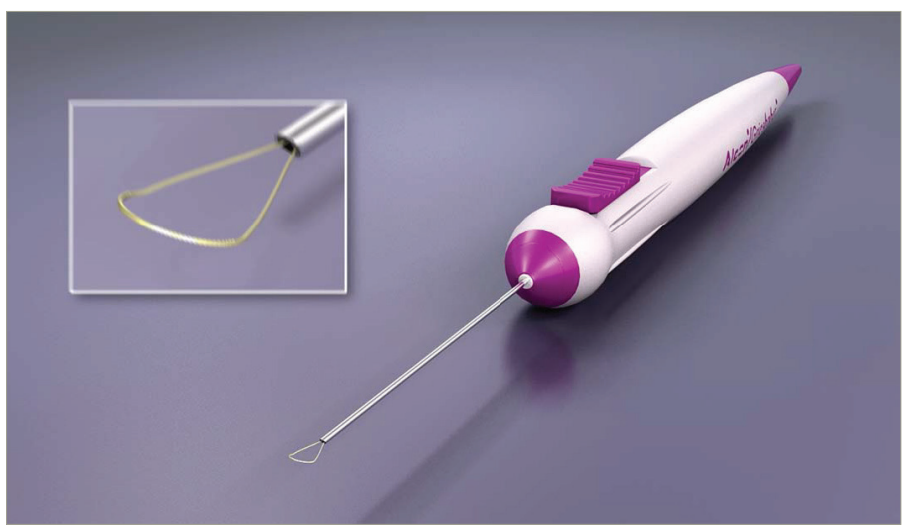

FINESSE ${ }^{\mathrm{TM}}$ Flex Loop (Alcon, Fort Worth, TX, US).

Figure 8: Three-dimensional printed customized retinal forceps: differences between surgeons' grip

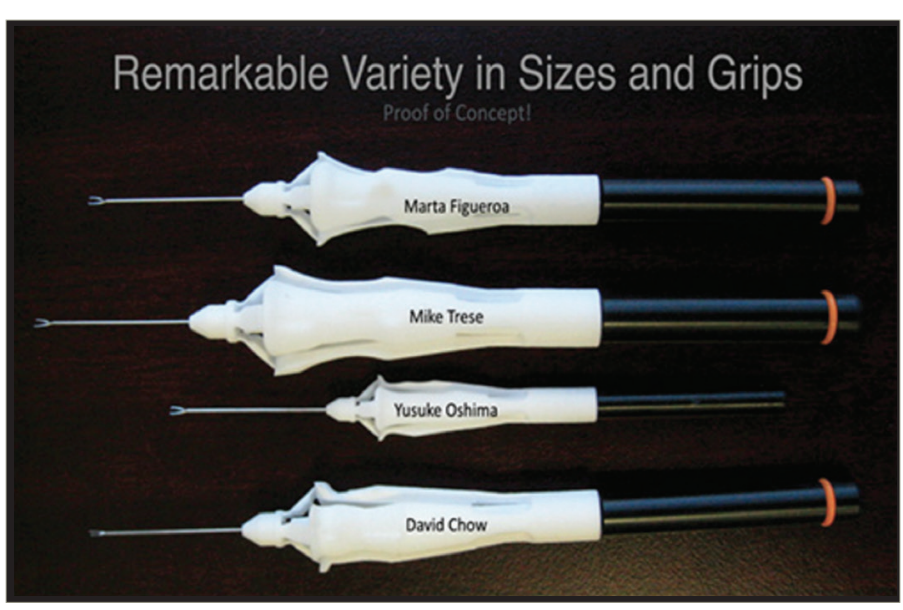

David Chow/Katalyst Surgical, LLC, Chesterfield, MO, US.

3D printing technology (David Chow/Katalyst Surgical, LLC, Chesterfield, $\mathrm{MO}$, US) takes all of these variables into consideration, giving surgeons customized forceps. After imprinting the surgeon's grip onto a mold, a 3D image of the grip is created and then superimposed to a forceps design which is then printed with a selective laser sintering 3D printer (Figure 8). ${ }^{4}$

\section{Sharkskin internal limiting membrane forceps}

The Sharkskin ${ }^{\mathrm{TM}}$ ILM forceps (Alcon, Fort Worth, TX, US) employs a new technology that increases friction on the backside of the ILM forceps' tip. Their microstructured tip improves grasping, resulting in less shredding and less need for regrasping during peeling. They were designed to facilitate initiation of ILM peeling (Figure 9). ${ }^{4}$

\section{Squeezer}

The Squeezer (Vitreq, Vierpolders, The Netherlands) is a disposable device used to obtain better control while injecting either dyes or perfluorocarbon liquids with a single-handed technique. It consists of a silicone tube within a plastic frame and it incorporates a Luer Lock for filling the silicon chamber and another to attach the silicon tip cannula (Figure 10). The silicone tube can be pre-filled by a nurse, and only by squeezing it with the thumb and forefinger, either a dye 
Figure 9: Sharkskin internal limiting membrane forceps
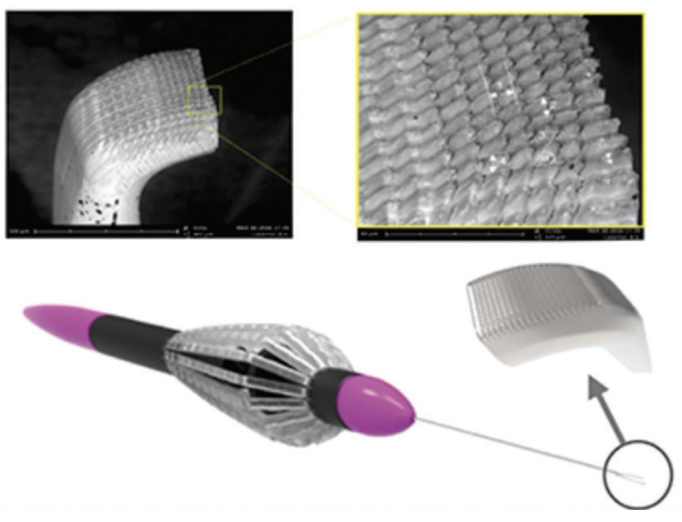

Sharkskin ${ }^{\mathrm{TM}}$ ILM forceps (Alcon, Fort Worth, TX, US). ILM = internal limiting membrane.

Figure 10: Squeezer

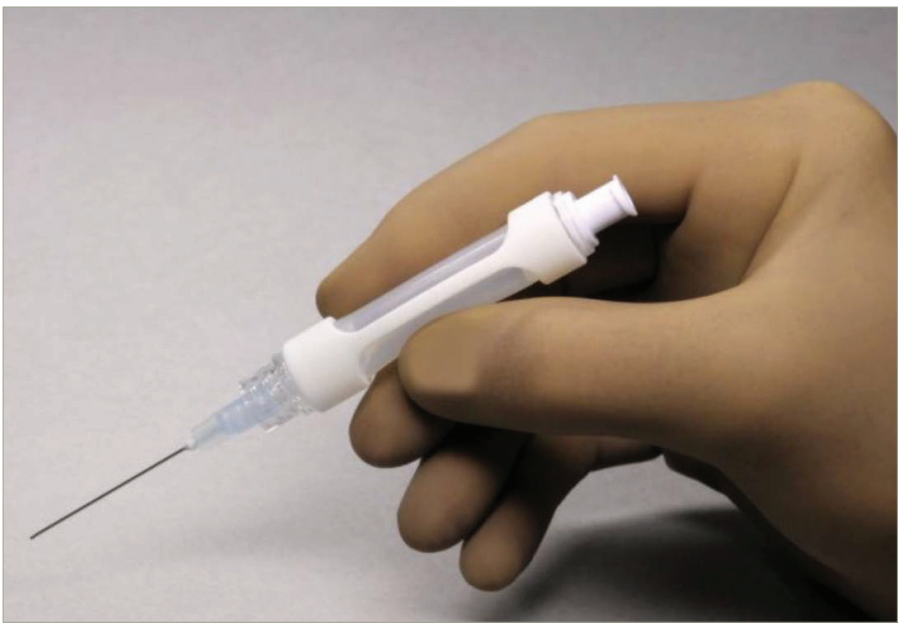

Squeezer (Vitreq, vierpolders, The Netherlands).

Figure 11: MicroDose injector

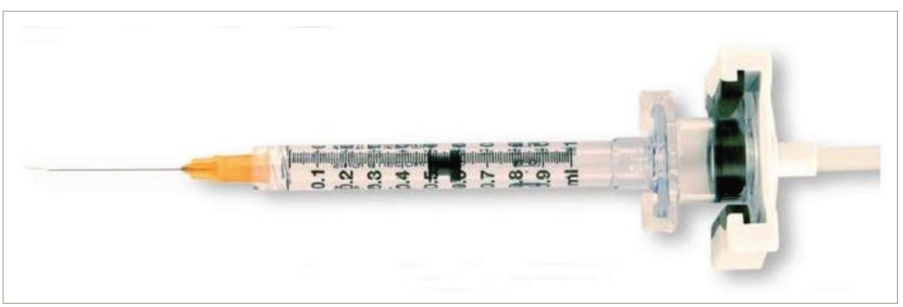

MicroDose ${ }^{\mathrm{TM}}$ injector (Medone Surgical, Inc., Sarasota, FL, US).

or perfluorocarbon liquid can be carefully delivered. The Luer Lock prevents backflow of vitreous once squeezing pressure is released.

\section{MicroDose injector}

The MicroDose ${ }^{\mathrm{TM}}$ injection kit (MedOne Surgical, Inc., Sarasota, FL, US) allows the surgeon to adapt the vitrectomy console viscous injection set to use a $1 \mathrm{ml}$ syringe (Figure 11).

Placing a subretinal injection cannula in a $1 \mathrm{ml}$ syringe will help the surgeon have absolute control while performing submacular surgery.
Figure 12: T-Tubes

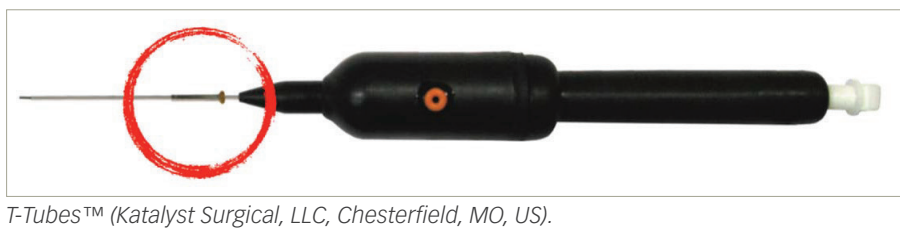

Figure 13: Intraoperative optical coherence tomography in a vitreomacular tractional syndrome

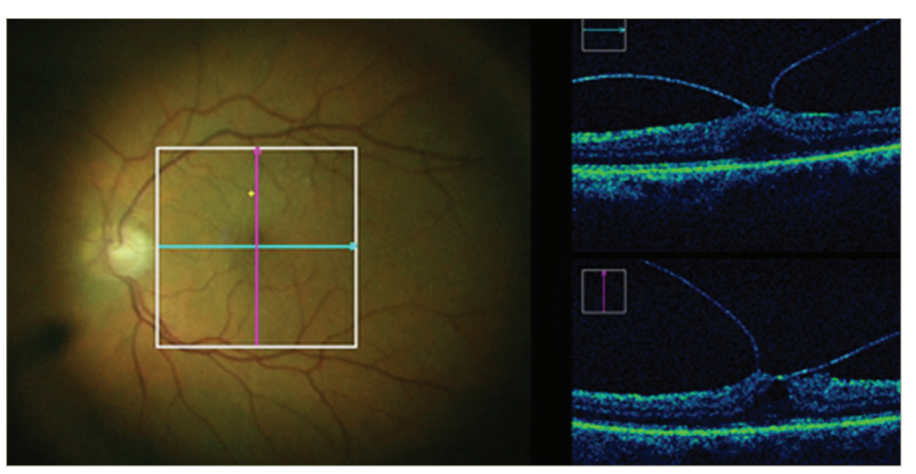

Figure 14: NGENUITY

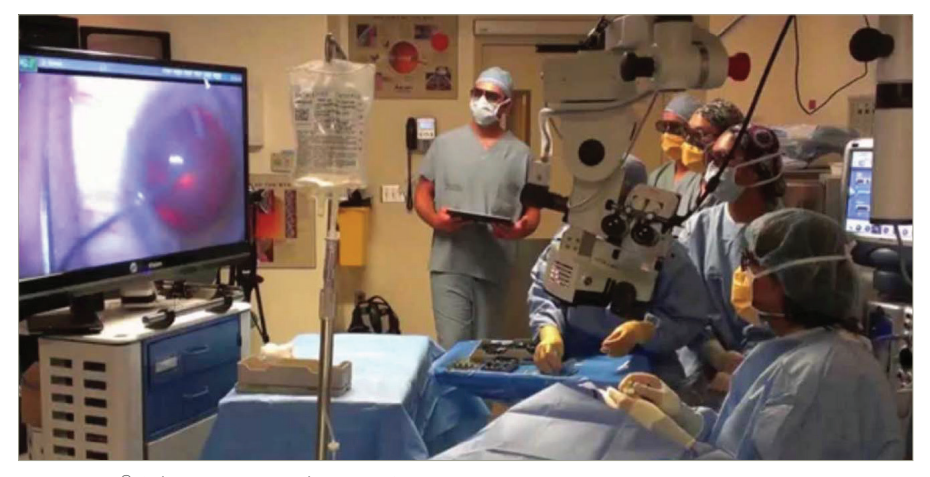

NGENUITY ${ }^{\circledast}$ (AlCon, Fort Worth, TX, US).

A MicroDose injector adaptor can be connected to the Constellation, Stellaris, and EVA platforms.

\section{T-Tube}

The advent of valved cannulae has improved the fluidics of vitrectomy by preventing aqueous reflux. However, the surgeon may experience difficulty introducing instruments through the valved cannula. To facilitate smooth insertion into valved cannulae, Katalyst developed a novel movable stainless-steel tube - the T-TubeTM (Katalyst Surgical, LLC, Chesterfield, MO, US) - with a diameter just larger than the cannula to be employed (Figure 12), improving the instrument's ability to pass through the valve, facilitating the insertion of instruments through valved cannulae.

\section{Intraoperative optical coherence tomography}

iOCT is currently one of the most revolutionary tools a retinal surgeon would like to have in the operating room. IOCT gains value in surgeries with poor visualization of the posterior pole and allows confirmation of surgical task completion such as ERM or ILM removal (Figure 13). ${ }^{2}$ 


\section{NGENUITY 3D Visualization System}

It was in the 1970s when Dr John P Baele Jr developed a revolutionary microscope that, combined with a television system, gave ophthalmologists the opportunity to perform, for the first time, "heads-up" surgery. The NGENUITY ${ }^{\circledR}$ 3D Visualization System (Alcon, Fort Worth, TX, US) is one of the latest and most revolutionary innovations developed for vitreoretinal surgery. It provides both "heads-up" surgery and a three-dimensional visualization system (Figure 14). This technology has been gaining acceptance in the field because of its well-known benefits, which include precise focus, improved depth of field, greater magnification, and increased safety from phototoxicity due to lower light levels during surgery.
The latest modification that has been made is integrated with the Constellation platform. The NGENUITY setup can now be customized and controlled either by a touch-panel screen or a foot pedal. Image mode access through the constellation allows surgeons to obtain snapshots, control camera orientation, or record the procedure. The interplay between light sources, filters, and image computer processing is receiving greater attention due to their ability to improve visualization and, as a consequence, surgery outcome. Current research studies are ongoing to evaluate and improve the potential of this visualization system. ${ }^{2} \square$

Machemer, R. A new concept for vitreous surgery. 2. Surgical techniques and complications. Am J Ophthalmol. 1972;74:1022-33.

2. Villegar VM, Murray TG. Know your retinal surgery toolbox - options abound for today's vitrectomy operating room. Retin Physician. 2018;15:24-9.

. de Oliveira PRC, Berger AR, Chow DR. Vitreoretinal instruments: vitrectomy cutters, endoillumination and wide-angle viewing systems. Int J Retin Vitr. 2016;2:28.

. Francisconi CLM, Chow DRC. What's new in vitreoretinal instrumentation? New designs, cut rates, and port migration aim to increase efficiency. Retin Phys. 2018;15:34-7.

5. SFGATE. John P. Beale Jr. 1999. Available at: www.sfgate.com/news/article/John-P-Beale-Jr-2944882.php (accessed August 30, 2018). 